University of Nebraska - Lincoln

DigitalCommons@University of Nebraska - Lincoln

\title{
A Channel Model for Wireless Underground Sensor Networks Using Lateral Waves
}

Xin Dong

University of Nebraska-Lincoln, xdong@cse.unl.edu

Mehmet C. Vuran

University of Nebraska-Lincoln, mcvuran@cse.unl.edu

Follow this and additional works at: https://digitalcommons.unl.edu/cseconfwork

Part of the Computer Sciences Commons

Dong, Xin and Vuran, Mehmet C., "A Channel Model for Wireless Underground Sensor Networks Using Lateral Waves" (2011). CSE Conference and Workshop Papers. 189.

https://digitalcommons.unl.edu/cseconfwork/189

This Article is brought to you for free and open access by the Computer Science and Engineering, Department of at DigitalCommons@University of Nebraska - Lincoln. It has been accepted for inclusion in CSE Conference and Workshop Papers by an authorized administrator of DigitalCommons@University of Nebraska - Lincoln. 


\title{
A Channel Model for Wireless Underground Sensor Networks Using Lateral Waves
}

\author{
Xin Dong and Mehmet C. Vuran \\ Cyber-Physical Networking Laboratory \\ Department of Computer Science \& Engineering \\ University of Nebraska-Lincoln, Lincoln, NE 68588 \\ Email: \{xdong, mcvuran\}@cse.unl.edu
}

\begin{abstract}
Wireless Underground Sensor Networks (WUSNs) are an emerging type of wireless sensor networks (WSNs), where sensor nodes are located under the ground and communicate through soil. The major challenge in the development of efficient communication protocols for WUSNs is the characterization of the underground channel. So far, none of the existing models fully capture all the components of electromagnetic signal propagation in the soil medium. In this paper, three major components that influence underground communication are identified: direct, reflected, and lateral waves, where the latter has not been analyzed for WUSNs so far. Accordingly, a closed-form three-wave (3W) channel model is developed based on EM propagation principles of signals through soil. The $3 \mathrm{~W}$ channel model is shown to agree well with both underground testbed experiments and EM analysis based on Maxwell's equations, which cannot be represented in closed-form.
\end{abstract}

\section{INTRODUCTION}

With the development of wireless sensor networks (WSNs), applications in constrained environments have gained considerable interest. One such area is wireless underground sensor networks (WUSNs), where the sensor motes are buried under soil and communicate with each other through soil. The novel applications of WUSNs include intelligent irrigation, environment monitoring, infrastructure monitoring, localization, and border patrol [1]. Especially in precision agriculture, WUSNs are envisioned to be a critical factor in improving water use efficiency by providing real-time information about soil properties [14].

For the design of WUSNs, an underground channel model, which captures the impacts of the soil medium on communication, is essential. Accordingly, the topology of the network, its communication protocols, and application parameters can be determined. Moreover, a channel model is critical for the evaluation of WUSN solutions. Compared to terrestrial WSNs, the lossy communication medium in WUSNs, which contains soil, air and water, incurs significantly higher attenuation. Moreover, the permittivity of the medium changes over time and space according to soil moisture [11], [13]. Thus, the established channel models for over-the-air communication cannot be directly applied to underground situations. We have developed a two-path underground channel model for WUSNs in [9], [17]. However, this model does not capture lateral waves, which manifest themselves in shallow deployments. In this work, we provide a closed-form channel model for underground communication that captures this specific phenomenon and validate it with experimental and analytical results.

The deployment of WUSNs is generally limited to depths of less than $50 \mathrm{~cm} \mathrm{[2],} \mathrm{[14].} \mathrm{In} \mathrm{these} \mathrm{cases,} \mathrm{a} \mathrm{portion} \mathrm{of} \mathrm{the}$ transmitted EM waves travel from soil to air, propagate along the soil-air interface, and thereafter penetrate the soil again and reach the receiver. These EM waves are called lateral waves [8], which are a major component of underground communication. Thus, for the modeling of WUSN communication channel, the propagation of the lateral waves must be included.

In [8], an EM field analysis of underground communication is provided using Maxwell's equations [8]. However, this model is computationally complex and difficult to use in practical applications, such as on-board channel evaluation by the sensor motes or large-scale simulations. In this paper, we develop a closed-form three-wave $(3 W)$ underground channel model to capture the characteristics of underground communication based on EM principles. The $3 \mathrm{~W}$ model is compared with our earlier two-path channel model in [17] and validated through testbed experiments in [13].

The rest of the paper of organized as follows: First, related work is discussed in Section II. In Section III, dielectric properties of soils and the relation between soil permittivity and soil moisture are introduced as well as the EM analysis of the underground communication. The developed closed-form underground channel model is described in detail in Section IV. Testbed validations and numerical evaluations of the model are presented in Section V. Finally, concluding remarks are provided in Section VI.

\section{RELATED WORK}

Wireless sensor communication in soil medium is a recently evolving field and there exist limited number of models to capture this phenomenon. In [9], [17], we developed a twopath channel model for WUSNs to capture the direct and reflected paths between underground sensors. Moreover, multipath fading is modeled as Rayleigh fading. However, the lateral wave component of the EM field, which manifests itself when the transceivers are buried near surface, is not considered. In [3] and [16], models for underground-to-aboveground communication are developed. These models, however, do not capture wireless communication between two underground nodes. Channel modeling for underground mines and road tunnels 


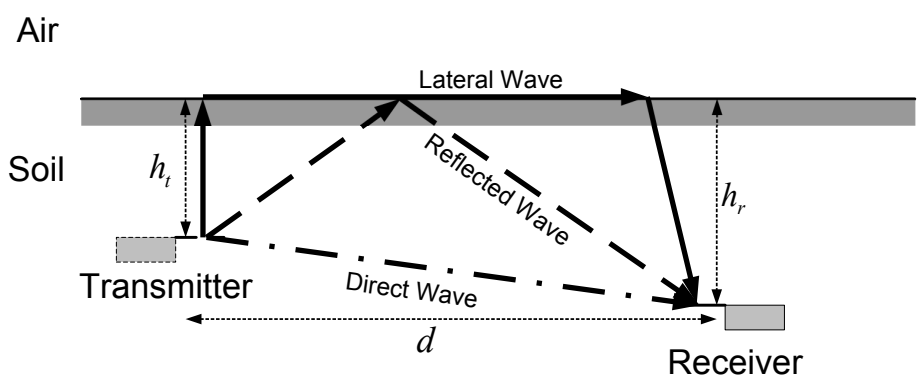

Fig. 1: The three electromagnetic waves at an underground point.

has been studied in [15]. Even though communications in underground mines and tunnels differ from terrestrial applications, the communication still takes in place in air and these models cannot capture propagation through soil. In addition, lateral waves are not observed for communication in mines and tunnels.

A complete description of the EM field in underground-tounderground communication is provided in [8]. The analysis is based on Maxwell's equations and can be broken down into three components: the direct wave, the reflected wave and the lateral wave. The resulting equations, however, are not in closed-form and hence, it is computationally intensive to utilize this model. Furthermore, the model in [8] is an approximation for far-field communication and is not accurate for near-field communication, which is common in WUSNs due to the high attenuation of soil. In this paper, we employ these equations as foundations to develop a closed-form channel model.

\section{BACKGROUND}

In this section, we discuss fundamental properties of soil and EM propagation in soil, which constitute the basis of our analysis.

\section{A. Dielectric Properties of Soil}

Wireless underground communication in soil is significantly affected by soil properties and their dynamics. Hence, it is imperative to capture the relative permittivity of soil-water mixture, which is impacted by several factors, such as soil bulk density, soil composition, soil moisture (Volumetric Water Content), salinity, and temperature. A large number of models have been proposed in literature to capture the characteristics of the relative permittivity [4], [10]. These models capture the relative permittivity of different components of soil-water mixture, namely, soil, air, free water and bounded water [4].

We utilize a semi-empirical dielectric model for soil in [10], which is well-suited for the frequency range of $0.3-1.3 \mathrm{GHz}$, a communication frequency band used in our system. This model is also employed in [17].

\section{B. Electromagnetic Field Analysis in Subsurface Soil}

The electromagnetic (EM) field model for underground wave propagation in [8] is derived from Maxwell's equations using Fourier transform techniques. In the following, we provide a brief explanation of the analysis approach and refer the reader to [8] or [5] for details. Considering an infinitesimal dipole buried at the subsurface of the soil as the transmitting antenna, the six components of the EM field at an observation location in the soil can be derived. These components are expressed in the form of integrations of Bessel functions. Based on the EM field, the time-average Poynting vector, which is the power density at a point, can be obtained as

$$
\mathscr{P}_{a v}=\frac{1}{2} \operatorname{Re}\left[\hat{\mathbf{E}} \times \hat{\mathbf{H}}^{*}\right],
$$

where $\hat{\mathbf{E}}$ is the electric field intensity and $\hat{\mathbf{H}}^{*}$ is the conjugate of the magnetic field intensity. The details of the derivations are provided in [8] and also presented in [5].

\section{Closed-Form Underground Channel Model}

The EM analysis for underground-to-underground communication in [8] is computationally complex. This approach is not suitable for practical applications where fast evaluation of the channel is needed. Especially in WUSNs, computation capacity-limited motes require a simpler way to estimate the channel quality from local sensor measurements to dynamically determine transmission power. In this section, we provide a channel model based on the analysis of the EM field and Friis equations. The model is in closed-form and has a good approximation as we report in Section V.

We consider the model depicted in Fig. 1, where a transmitter and a receiver are located at a distance of $d$ and depths $h_{t}$ and $h_{r}$, respectively. The underground communication link between this pair consists of three EM waves: (1) The direct wave is the result of line-of-sight propagation between the transmitter to the receiver through soil. (2) The reflected wave is reflected by the air-soil interface that also propagates through soil. (3) The lateral wave propagates in air and penetrates back to soil. In the following, the Poynting vectors for the three components are derived. Then, for a specific antenna model, the derived Poynting vectors are used to find the received power. This leads to a closed-form $3 \mathrm{~W}$ channel model, that can be used to evaluate wireless underground communication.

\section{A. The Direct Wave}

The direct wave is the spherical wave traveling outward radially from the transmitter to the receiver in a line-of-sight path. In over-the-air communication, direct waves are the dominant part of the received power. However, in wireless underground 
communications, the direct wave is attenuated much faster in especially wet soils due to soil conductivity. Nonetheless, the direct wave can still be modeled based on the well-known Friis equations. The time-average Poynting vector of the direct wave is

$$
\mathscr{P}_{a v}^{d}=\left(\frac{D_{d}}{4 \pi r_{1}^{2}}\right) e^{i 2 k_{s} r_{1}} G_{t},
$$

where $r_{1}$ is the distance between the source and the observation point; $k_{s}=\beta_{s}+i \alpha_{s}=\omega \sqrt{\mu_{0} \hat{\epsilon}_{s}}$ is the wave number in soil, where $\beta_{s}$ accounts for phase shifting and $\alpha_{s}$ accounts for attenuation; $\omega=2 \pi f$, where $f$ is the frequency of the wave; and $\mu_{0}$ and $\hat{\epsilon}_{s}$ are the permeability and permittivity of the soil. We assume the soil is nonmagnetic, thus, the permeability of vacuum, $\mu_{0}$, is employed. In (2), $G_{t}$ is the antenna gain of the transmitter and $D_{d}$ is a constant related to soil permittivity, which will be discussed in Section V. The component $e^{i 2 k_{s} r_{1}}$ accounts for the attenuation and phase shifting of wave propagation in soil. The attenuation is due to the fact that $k_{s}$ is a complex number instead of a real number. Note that (2) is different from Friis equation because it only describes the power density at a location, and the receiving area of the receive antenna is not considered. To calculate the received power, the Poynting vector is multiplied by the receiving area, which is defined as [7]: $A=\lambda_{s}^{2} G_{r} / 4 \pi$, where $\lambda_{s}$ is the wavelength in soil and $G_{r}$ is the antenna gain of the receiver. Based on Fig. 1, $r_{1}=\sqrt{\left(h_{t}-h_{r}\right)^{2}+d^{2}}$ and the wavelength in soil is calculated as $\lambda_{s}=2 \pi / \beta_{s}$.

The direct gain of an antenna depends on its propagation pattern. For the purpose of comparing with the EM analysis, an infinitesimal dipole is assumed, with a direct gain of $G_{t}=\sin \alpha$ , where $\alpha$ is the angle related to the extension line of the dipole.

\section{B. The Reflected Wave}

Due to the different dielectric constants of air and soil, the traveling wave incident to the air-soil interface is reflected as shown in Fig. 1. For the tractability of the analysis, the air-soil interface is assumed to be flat. Thus, time-average Poynting vector of the reflected wave is

$$
\mathscr{P}_{a v}^{r}=D_{r}\left(\frac{1}{4 \pi r_{2}^{2}}\right) e^{i 2 k_{s} r_{2}} G_{t} \Gamma^{2},
$$

where $D_{r}$ is a constant related to soil permittivity, $r_{2}$ is the length of the reflection path, $r_{2}=\sqrt{\left(h_{t}+h_{r}\right)^{2}+d^{2}}$, and $\Gamma$ is the reflection coefficient given by [7]:

$$
\Gamma=\frac{\frac{1}{n} \cos \theta_{r i}-\cos \theta_{r t}}{\frac{1}{n} \cos \theta_{r i}+\cos \theta_{r t}},
$$

where $n$ is the refractive index of soil, $\theta_{r i}$ and $\theta_{r t}$ are the incident angle and the refracted angle, respectively. Based on Snell's law,

$$
\begin{aligned}
\sin \theta_{r i} & =\frac{d}{r_{2}}, & \cos \theta_{r i} & =\frac{h_{t}+h_{r}}{r_{2}}, \\
\sin \theta_{r t} & =n \sin \theta_{r i}, & \cos \theta_{r t} & =\sqrt{1-\sin ^{2} \theta_{r t}} .
\end{aligned}
$$

Since the permittivity of soil is a complex number, the refractive index of soil is calculated as

$$
n=\sqrt{\frac{\sqrt{\epsilon^{\prime 2}+\epsilon^{\prime \prime 2}}+\epsilon^{\prime}}{2}},
$$

in which $\epsilon^{\prime}$ and $\epsilon^{\prime \prime}$ are the real and imaginary parts of the relative permittivity of the soil.

For the attenuation and phase shifting, since the whole path of the reflected wave is still in soil, only the wave number in soil, $k_{s}$, is employed.

\section{The Lateral Wave}

In the previous underground-to-underground channel models, only direct waves and reflected waves have been considered. However, due to the fact that the sensor motes are buried near the air-soil interface, lateral waves are one of the tree major components of the EM field [8], and also dominate communication in the far field. The path of the lateral wave is shown in Fig. 1. From soil to air, the wave travels vertically from the transmitter to the interface. At the interface, it propagates horizontally along the interface as a spherical wave. At an incidence angle of $\sim \pi / 2$, the wave penetrates into the soil. The refracted wave is a portion of the original wave and it travels downward from the air-soil interface to the receiver in soil. The corresponding time-average Poynting vector of the lateral wave is

$$
\mathscr{P}_{a v}^{L}=\left(\frac{D_{l}}{4 \pi d^{4}}\right) e^{i 2 k_{s}\left(h_{t}+h_{r}\right)} e^{i 2 k_{0} d} T^{2} G_{t},
$$

where $T$ is the refraction coefficient when the wave travels from air to soil and $D_{l}$ is a constant for the diffusion along air-soil interface. Note, the refraction coefficient from soil to air is not considered since all the energy is refracted to the air. However, from air to soil, only part of the wave is refracted to the soil, thus $T$ is less than 1 and is defined as

$$
T=\frac{2 \cos \theta_{l i}}{n \cos \theta_{l i}+\cos \theta_{l t}},
$$

where $n$ is the refractive index of the soil as shown in (6).

Since the motes are very close to soil-air interface, the diffusion in the soil is negligible. Thus, the diffusion is only related to the horizontal distance of the transceiver pair. Also, the diffusion cannot be modeled as inversely proportional to $d^{2}$ because of the interface. Based on the EM wave analysis, both the $\mathbf{E}$ field and the $\mathbf{H}$ field of the lateral wave are proportional with $1 / d^{2}$. Thus, the power density, which is a product of $\mathbf{E}$ and $\mathbf{H}$, is proportional to $1 / d^{4}$. Therefore, $d^{4}$ is used in (7) and a constant $D_{l}$ is employed.

The lateral wave path consists of two portions in soil and one portion in air. Accordingly, the attenuation and phase shifting consists of two parts in (7): $e^{i 2 k_{0} d}$ is the attenuation and phase shifting in the air, while $e^{i 2 k_{s}\left(h_{t}+h_{r}\right)}$ is that in the soil. Due to the high refractive index of soil, the refraction angle is quite small. For volumetric water content (VWC) in the range of $10 \%-40 \%$, the refraction angle is in the range of $10^{\circ}-20^{\circ}$. Therefore, the wave path length in the soil is approximated by the sum of the burial depths of the transmitter and the receiver. 


\section{The Composed Field}

The total power density $\mathscr{P}_{a v}$ can then be derived as the superposition of the three components. Namely,

$$
\mathscr{P}_{a v}=\mathscr{P}_{a v}^{d}+\mathscr{P}_{a v}^{r}+\mathscr{P}_{a v}^{L} .
$$

Note the sum is a vector sum since the three components have different directions as shown in Fig. 1. To calculate the received power of a receiving antenna, power density is multiplied by the receiving area of a specific antenna. Here, to compare with the EM field analysis, we map the total power density into Cartesian coordinates,

$$
\begin{aligned}
& \mathscr{P}_{a v}^{x}=\left(\mathscr{P}_{a v}^{d} \times \frac{d}{r_{1}}+\mathscr{P}_{a v}^{r} \times \frac{d}{r_{2}}+\mathscr{P}_{a v}^{L} \times \sin \theta_{l t}\right) \cos \phi, \\
& \mathscr{P}_{a v}^{y}=\left(\mathscr{P}_{a v}^{d} \times \frac{d}{r_{1}}+\mathscr{P}_{a v}^{r} \times \frac{d}{r_{2}}+\mathscr{P}_{a v}^{L} \times \sin \theta_{l t}\right) \sin \phi, \quad(10) \\
& \mathscr{P}_{a v}^{z}=\mathscr{P}_{a v}^{d} \times \frac{\left|h_{t}-h_{r}\right|}{r_{1}}+\mathscr{P}_{a v}^{r} \times \frac{h_{t}+h_{r}}{r_{2}}+\mathscr{P}_{a v}^{L} \times \cos \theta_{l t},
\end{aligned}
$$

where $\phi$ is the azimuth angle of the receiver in the cylindrical coordinates.

\section{E. The Received Power of an Isotropic Antenna Pair}

In traditional channel models, isotropic antennas are assumed [7]. In this section, we derive the received power when isotropic antennas are used for transmitting and receiving. For isotropic antennas, $G_{t}=G_{r}=1$. Thus, the receiving area becomes $A_{\text {iso }}=\lambda_{s}^{2} / 4 \pi$.

The three components of the received power, written in logarithmic form, are

$$
\begin{aligned}
P_{r}^{d}= & P_{t}+20 \log _{10} \lambda_{s}-20 \log _{10} r_{1}-8.69 \alpha_{s} r_{1}-45 \\
P_{r}^{r}= & P_{t}+20 \log _{10} \lambda_{s}-20 \log _{10} r_{2}-8.69 \alpha_{s} r_{2} \\
& +20 \log _{10} \Gamma-45 \\
P_{r}^{L}= & P_{t}+20 \log _{10} \lambda_{s}-40 \log _{10} d-8.69 \alpha_{s}\left(h_{t}+h_{r}\right) \\
& +20 \log _{10} T-30,
\end{aligned}
$$

where $\Gamma$ and $T$ are given in (4) and (8), respectively, $\alpha_{s}$ is the imaginary part of the wave number in soil and $\lambda_{s}$ is the wavelength in soil. For isotropic antenna, the overall received power is the sum of the three components. Thus,

$$
P_{r}=10 \log _{10}\left(10^{\frac{P_{r}^{d}}{10}}+10^{\frac{P_{r}^{r}}{10}}+10^{\frac{P_{r}^{L}}{10}}\right) .
$$

\section{Model COMPARISON AND VERIFICATION}

In this section, we compare the developed 3-wave model (3W model) with the EM field analysis [8] to show its performance of approximation. In addition, the model is also compared with the two-path model [9], [17] and testbed results for verification.

\section{A. Comparison with Electromagnetic Analysis}

A comparison of the EM field analysis and the closed-form $3 \mathrm{~W}$ model is shown in Fig. 2, where an approximation of the EM field from [8] is used. Two values of volumetric water content (VWC) are assumed: (1) dry soil with VWC at 10\%, and (2) wet soil with VWC at $35 \%$. The VWC values are from field measurements as reported in [6]. For each of the situations,

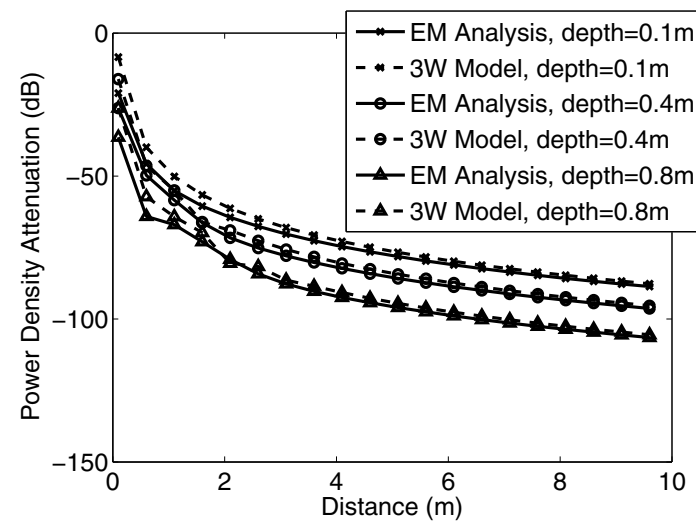

(a) $\mathrm{VWC}=10 \%$

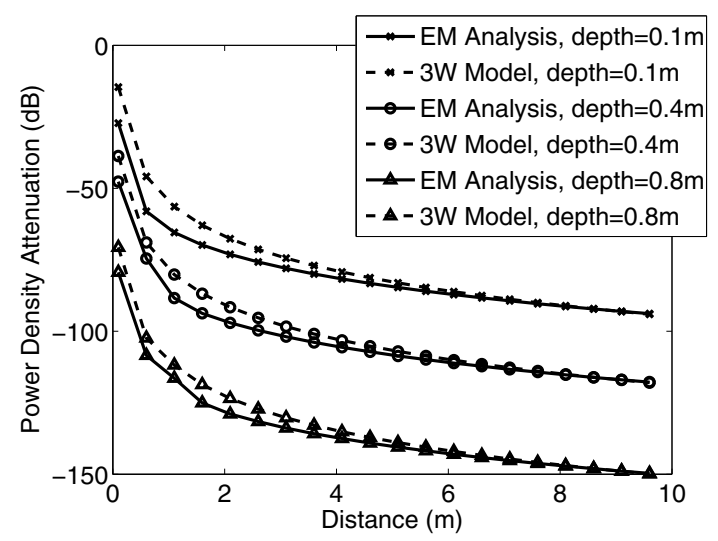

(b) $\mathrm{VWC}=35 \%$

Fig. 2: Comparison of the results of the electromagnetic analysis and the $3 \mathrm{~W}$ model with different burial depths.

three burial depths, $0.1 \mathrm{~m}, 0.4 \mathrm{~m}$ and $0.8 \mathrm{~m}$, are investigated. The figures show the attenuation of the underground channel versus horizontal distance between the transmitter and the receiver.

To apply the $3 \mathrm{~W}$ model, the values of $D_{d}, D_{r}$ and $D_{l}$ need to be determined. Ideally, those values should be obtained through extensive empirical evaluations. Here, we compare our model to the EM model, and employ minimum mean square error (MMSE) to estimate those values, which are found to be $D_{d}=$ $D_{r}=0.005$ and $D_{l}=0.15$.

It is observed that the $3 \mathrm{~W}$ model captures the main components of the EM field and matches the results of the analysis. Furthermore, the two models match better at the far field than the near field. For the near field $(d<1 \mathrm{~m})$ the results of the $3 \mathrm{~W}$ model is about $10 \%$ higher than the EM model. However, for the far field $(d>2 \mathrm{~m})$, the results are less than $5 \%$ higher. For the very far field $(d>6 \mathrm{~m})$, the difference of the two models is less than $1 \%$. This is mainly because the EM analysis is an approximation for the far field and for the near field, it has lower accuracy. Thus, these two models need to be compared with detailed testbed results.

The effects of the soil moisture and burial depth can also be analyzed from the figures. When the transceivers are deeply 


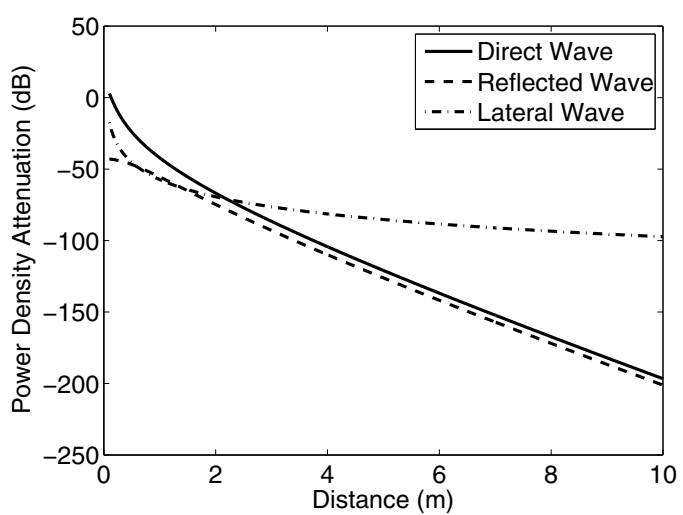

(a)

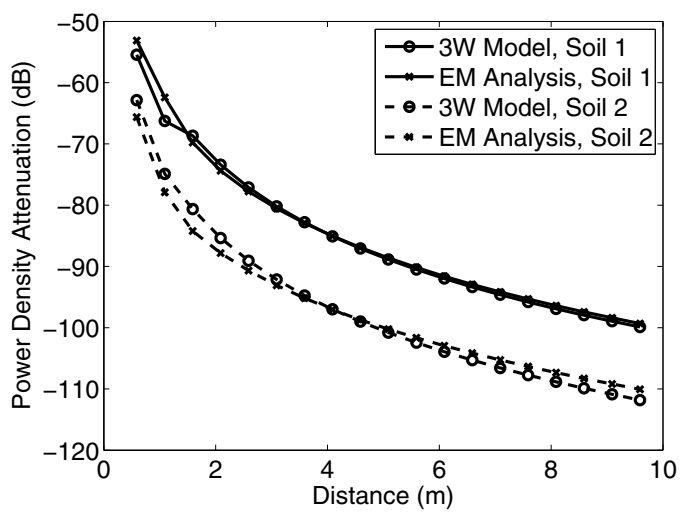

(b)

Fig. 3: Analysis of the Model. (a) The comparison of the three components over distance; (b) application of the model to two soil types.

buried, the path in the soil increases and the attenuation increases. Accordingly, when the VWC is $10 \%$ and the motes are buried at $0.1 \mathrm{~m}$ (Fig. 2(a)), the attenuation at the distance of $4 \mathrm{~m}$ is $-72 \mathrm{~dB}$. At the same horizontal distance, the attenuation increases to $-80 \mathrm{~dB}$ when the motes are buried at $0.4 \mathrm{~m}$ and to $-91 \mathrm{~dB}$ if they are buried at $0.8 \mathrm{~m}$.

Another factor that influences underground communication is the soil moisture. High soil moisture increases the attenuation of the EM waves and decreases the signal strength. As shown in Fig. 2(a), for a burial depth of $0.4 \mathrm{~m}$ and a VWC of $10 \%$, the signal attenuation at a distance of ), $2 \mathrm{~m}$ is $70 \mathrm{~dB}$. On the other hand, if the VWC increases to $35 \%$ (Fig. 2(b)), the attenuation at $2 \mathrm{~m}$ increases to $90 \mathrm{~dB}$. Moreover, the effect of the soil moisture varies by burial depth. If the motes are buried shallow $(0.1 \mathrm{~m})$, the soil moisture effect is almost negligible. This occurs because the lateral wave path in soil is very short, and hence, the attenuation by soil has a small impact. Conversely, if the motes are buried deep, the effect of the soil moisture is clearly observed. As an example, at a burial depth of $0.8 \mathrm{~m}$, an increase in VWC from $10 \%$ to $35 \%$ leads to an increase in attenuation by more than $30 \mathrm{~dB}$.

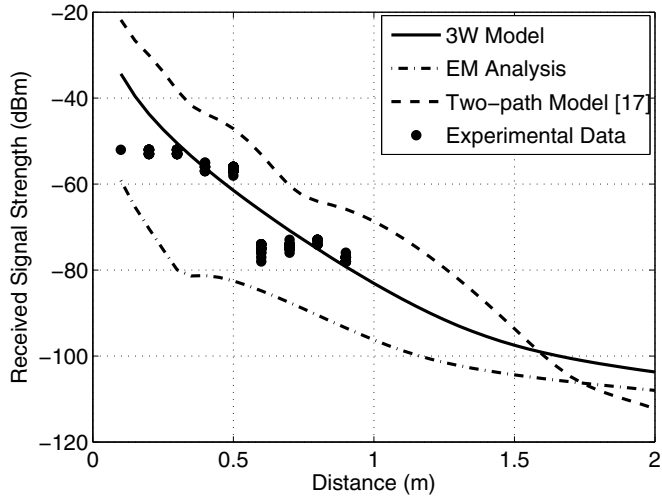

Fig. 4: The comparison of the Proposed Model, the Two-path Model and Testbed Results. (burial depth=0.4 m)

\section{B. Model Analysis}

In Fig. 3(a), the power density of three components, the direct wave, the reflected wave and the lateral wave, over distance are depicted. The transmitter is buried at the depth of $0.4 \mathrm{~m}$ and the receiver is buried at the depth of $0.5 \mathrm{~m}$. It is shown that the power density of the reflected wave is about $10 \mathrm{~dB}$ lower than the direct wave in the near field but close to the direct wave in the far field since at the far field the path lengths of the two waves are similar. More importantly, at distance less than $2 \mathrm{~m}$, the direct wave has a higher power density than the lateral wave. Yet, for longer distances, the direct wave and the reflected wave are attenuated drastically, such that the lateral wave becomes dominant. For instance, at the distance of $3 \mathrm{~m}$, the density of the direct wave is $10 \mathrm{~dB}$ lower than the lateral wave, while at the distance of $4 \mathrm{~m}$ it is $20 \mathrm{~dB}$ lower.

We also apply the $3 \mathrm{~W}$ model to two different soil types.

- Soil 1: Dry sandy soil with a percentage of sand, $S=$ $50 \%$, a percentage of clay, $C=15 \%$ and $\mathrm{VWC}=5 \%$ is considered.

- Soil 2: This soil from our testbed, which contains $31 \%$ of sand, $29 \%$ of clay and $\mathrm{VWC}=20 \%$.

The channel qualities for these two soil types are shown in Fig. 3(b). For each soil type, we compare the $3 \mathrm{~W}$ model with the EM analysis. It is shown that for both soil types, $3 \mathrm{~W}$ model agrees well with the EM analysis. Similar to the results in Section V-A, in the near field $(d<1 \mathrm{~m})$, the $3 \mathrm{~W}$ model results in an error of $10 \%$ compared to the EM analysis, and in the far field $(d>2 \mathrm{~m})$, the difference reduces to less than $5 \%$. Moreover, Soil 1 has a lower attenuation $(10 \mathrm{~dB})$ than Soil 2. This is mainly because the sandy soil is less capable of holding water, a primary factor of wave attenuation.

\section{Comparison with the Two-path Model and Empirical Re-} sults

The results of the $3 \mathrm{~W}$ model, the two-path model [17] and testbed measurements are shown in Fig. 4. The testbed results are from our previous work with Mica2 sensor motes in [13], which are buried at the depth of $0.4 \mathrm{~m}$ and the transmission 
power is set to $10 \mathrm{dBm}$. For each of the horizontal distance, 50 samples are obtained and depicted in the figure. The result of the $3 \mathrm{~W}$ model is calculated using (12). The properties of the soil, such as the percentage of sand, $S=31 \%$, the percentage of the clay, $C=29 \%$, are taken from the testbed as the input to the two models.

Due to the limitation of Mica2 motes, the signal strength measurement is not accurate, especially when the received power is higher than $-50 \mathrm{dBm}$, where clipping effect is observed [12]. Nevertheless, it is still clearly shown that the $3 \mathrm{~W}$ model is more accurate than the two-path model. Comparing the results at $0.3-0.9 \mathrm{~m}$, except the results at $0.6 \mathrm{~m}$, the difference between $3 \mathrm{~W}$ model and the experiment results is less than $3 \mathrm{~dB}$. The experiment data at $0.6 \mathrm{~m}$ is irregularly low, which may be caused by low quality of the specific mote or other environment factors. The results from the two-path model is $10-15 \mathrm{~dB}$ higher than the experiment results. As mentioned in Section V-A, the approximation of the EM analysis has low accuracy in the near field, which causes the results to be $10-20 \mathrm{~dB}$ lower than the experiment results. These initial comparisons illustrate the accuracy of the model but we also acknowledge the need for further testbed experiments with different soil types to fine tune the model, which is out of the scope of this work.

\section{CONCLUSIONS}

In this paper, we develop a closed-form three-wave $(3 \mathrm{~W})$ channel model for communication in soil medium. Compared to previous underground channel models, the model includes all the three components in the field, namely, the direct wave, the reflected wave and more importantly, the lateral wave. Validations with numerical analysis and empirical channel measurements show that the $3 \mathrm{~W}$ model is a good approximation to the electromagnetic analysis and agrees well with testbed experiments.

\section{ACKNOWLEDGEMENTS}

This work is supported by an NSF CAREER award (CNS0953900), USGS (2010NE209B), and UNL Water Center. The authors would like to thank Dr. Suat Irmak for his valuable comments throughout the development of the experiments and William Rathje for his support during the experiments at Clay Center.

\section{REFERENCES}

[1] I. F. Akyildiz and E. P. Stuntebeck, "Wireless underground sensor networks: Research challenges," Ad Hoc Networks Journal (Elsevier), vol. 4, pp. 669-686, July 2006.

[2] H. R. Bogena, M. Herbst, J. A. Huisman, U. Rosenbaum, A. Weuthen, and $\mathrm{H}$. Vereecken, "Potential of wireless sensor networks for measuring soil water content variability," Vadose Zone Journal, vol. 9, no. 4, pp. 1002-1013, November 2010.

[3] H. R. Bogena, J. A. Huismana, H. Meierb, U. Rosenbauma, and A. Weuthena, "Hybrid wireless underground sensor networks: Quantification of signal attenuation in soil," Vadose Zone Journal, vol. 8, no. 3, pp. 755-761, August 2009.

[4] M. Dobson, F. Ulaby, M. Hallikainen, and M. El-Rayes, "Microwave dielectric behavior of wet soil—part ii: Dielectric mixing models," IEEE Transactions on Geoscience and Remote Sensing, vol. GE-23, no. 1, pp. $35-46$, January 1985.

[5] X. Dong and M. C. Vuran, "A channel model for wireless underground sensor networks using lateral waves," Dept. of Computer Science \& Engineering, Univ. of Nebraska-Lincoln, Tech. Rep. TR-UNL-CSE-20100011, 2011 .

[6] S. Irmak, "Nebraska water and energy flux measurement, modeling, and research network (NEBFLUX)," Trans. ASABE, vol. 53, no. 4, pp. $1097-$ 1115, July 2010.

[7] C. T. Johnk, Engineering Electromagnetic Fields and Waves, 2nd ed. John Wiley \& Sons, 1988.

[8] R. King, M. Owens, and T. Wu, Lateral Electromagnetic Waves. Springer-Verlag, 1992.

[9] L. Li, M. Vuran, and I. Akyildiz, "Characteristics of underground channel for wirless underground sensor networks," in Proc. of IFIP Mediterranean Ad Hoc Networking Workshop (Med-Hoc-Net '07), Corfu, Greece, June 2007.

[10] N. Peplinski, F. Ulaby, and M. Dobson, "Dielectric properties of soil in the 0.3-1.3 ghz range," IEEE Transactions on Geoscience and Remote Sensing, vol. 33, no. 3, pp. 803-807, May 1995.

[11] A. R. Silva and M. C. Vuran, "Communication with aboveground devices in wireless underground sensor networks: An empirical study," in Proc. of IEEE International Conference on Communications (ICC '10), Cape Town, South Africa, May 2010, pp. 1-6.

[12] — - "Development of a Testbed for Wireless Underground Sensor Networks," EURASIP Journal on Wireless Communications and Networking, vol. 2010, 2010.

[13] —, "Empirical evaluation of wireless underground-to-underground communication in wireless underground sensor networks," in Proc. of IEEE International Conference on Distributed Computing in Sensor Systems (DCOSS '09), Marina del Rey, CA, June 2009, pp. 231-244.

[14] - , (CPS $)^{2}$ : integration of center pivot systems with wireless underground sensor networks for autonomous precision agriculture," in Proc. of ACM/IEEE International Conference on Cyber-Physical Systems (ICCPS '10), Stockholm, Sweden, April 2010, pp. 79-88.

[15] Z. Sun and I. Akyildiz, "Channel modeling and analysis for wireless networks in underground mines and road tunnels," IEEE Transactions on Communications, vol. 58, no. 6, pp. 1758-1768, June 2010

[16] _ - "Connectivity in wireless underground sensor networks," in Proc. of IEEE Communications Society Conference on Sensor Mesh and Ad Hoc Communications and Networks (SECON '10), Boston, MA, 2010.

[17] M. C. Vuran and I. F. Akyildiz, "Channel model and analysis for wireless underground sensor networks in soil medium," Physical Communication, vol. 3, no. 4, pp. 245-254, December 2010. 